Revue d'histoire de l'enfance " irrégulière »

Le Temps de l'histoire

18 | 2016

Le psychiatre, l'enfant et l'État

\title{
Le premier congrès international de Psychiatrie infantile (Paris, 1937), ou le baptême d'une spécialité ambitieuse
}

The First International Congress on Child Psychiatry (Paris, 1937): walking towards the consecration of an ambitious specialty

\section{Samuel Boussion}

\section{OpenEdition}

Journals

Édition électronique

URL : http://journals.openedition.org/rhei/3880

DOI : $10.4000 /$ rhei.3880

ISSN : $1777-540 \mathrm{X}$

Éditeur

Presses universitaires de Rennes

Édition imprimée

Date de publication : 30 novembre 2016

Pagination : $57-80$

ISBN : 978-2-7535-5175-6

ISSN : $1287-2431$

\section{Référence électronique}

Samuel Boussion, «Le premier congrès international de Psychiatrie infantile (Paris, 1937), ou le baptême d'une spécialité ambitieuse », Revue d'histoire de l'enfance «irrégulière » [En ligne], 18 | 2016, mis en ligne le 30 novembre 2018, consulté le 30 avril 2019. URL : http://journals.openedition.org/ rhei/3880 ; DOl : 10.4000/rhei.3880 


\section{Le premier congrès international de Psychiatrie infantile (Paris, 1937), ou le baptême d'une spécialité ambitieuse}

\begin{abstract}
Cet article propose de mettre en perspective le premier congrès international de Psychiatrie infantile. Organisé par Georges Heuyer du 24 juillet au 1er août 1937, dans le cadre de l'Exposition internationale de Paris, le congrès réunit plusieurs centaines de médecins venus de plus d'une vingtaine de pays. L'événement est d'importance et une lecture des archives d'Heuyer en fait ressortir ses multiples enjeux. D'abord, il repose sur les intentions professionnelles de ce dernier, tentant d'atteindre le firmament de sa carrière de psychiatre d'enfant. Ensuite pour le groupe professionnel dans son ensemble, qui par ce moment tente d'affirmer la légitimité de cette spécialité, la psychiatrie infantile, aux yeux des pouvoirs publics notamment. Le moment est d'importance, tant l'enfance déficiente est à l'ordre du jour politique sous le Front populaire. II est tout aussi majeur sur le plan international, alors que les circulations sont denses et que la psychiatrie devient un objet scientifique central. Enfin, le congrès international, moment de croisement par excellence, permet d'aborder les questions scientifiques mises à l'agenda des psychiatres et ainsi de saisir les angles d'étude de l'enfant. Enfin, le congrès international est un moment d'échanges par excellence et permet ainsi aux psychiatres de débattre sur les différentes approches pour analyser l'enfant dans sa complexité.
\end{abstract}

This article proposes to put into perspective the First International Congress of Child Psychiatry. Organised by Georges Heuyer from 24 July to 1 August 1937, as part of the International Exhibition in Paris, the conference brings together hundreds of doctors from over twenty countries. The event is important and an exploration of the Heuyer files underscores its multiple challenges. First, it is based on his professional intentions, trying to reach the firmament of his child psychiatrist career. Then for the professional group as a whole which is at that time trying to build up the legitimacy of this specialty of child psychiatry for the authorities especially. Handicapped children is an important issue at this time given that it is at the forefront of the political agenda of the Popular Front' cabinet. It is equally important at the international level, since the circulations are intense and psychiatry is becoming a central scientific object. Finally, the international congress is a time of exchanges by excellence and drives psychiatrists to discuss over the different approaches raised to explore and to understand the complexity of the child.

\author{
Samuel Boussion \\ Maître de conférences \\ en sciences de l'édu- \\ cation, Clrceft, Heduc, \\ université Paris 8 \\ Saint-Denis.
}


1. Selon les termes de la convention de Paris de 1928.

2. Archives nationales (AN), Enseignement, CNRS; commissariat général du Salon des arts ménagers, expositions et salons, 19850025/123: WURMSER Jean, «L'Exposition de 1937 doit être un acte de foi ", Le Parisien, jeudi 28 février 1935.

3. An, Présidence du Conseil, F/60/9691937. Ce que sera l'Exposition internationale de Paris, brochure, 1935.

4. Hauser Claude, Valloton François (dir.), Dossier « Les expositions internationales, mises en scène de la modernité ", Relations internationales, 164, 4, 2015.
Mots-clés: psychiatrie infantile, Georges Heuyer, congrès, hygiène mentale, exposition internationale

Keywords : child psychiatry, Georges Heuyer, congress, mental hygiene, international exposition

Z ntre mai et novembre 1937, Paris se transforme en capitale mondiale Cà l'occasion de l'Exposition internationale organisée par la France et dédiée cette année-là aux "Arts et techniques dans la vie moderne ". Même si elle n'est qu'une exposition de seconde catégorie et ne prétend donc pas être " universelle ${ }^{1}$ », elle est portée par de grands desseins. Pour un pays qui ressent encore les effets d'une profonde crise économique, elle est présentée comme un carrefour des peuples et comme une occasion de sortir la France de son marasme en créant une atmosphère d'activité spirituelle et matérielle, de remédier au chômage, de ranimer le tourisme, bref d'opérer une " expérience de galvanisation ", selon les mots de Paul Léon, commissaire adjoint de l'Exposition en $1935^{2}$. À plusieurs reprises, ses promoteurs ravivent les souvenirs glorieux des expositions passées, celles tenues à Paris en 1889 et 1900, l'Exposition des Arts décoratifs, en 1925, plus récemment l'Exposition universelle de Chicago de 1933-1934, connue sous le nom de Century of Progress, situant l'Exposition dans une filiation qui vise la synthèse entre art, pensée et technique, se déployant comme une "Exposition de la civilisation ${ }^{3}$ ", rien de moins.

Alors, quand celle-ci s'ouvre en mai 1937, les différents pavillons édifiés dans la zone d'exposition, mais aussi les œuvres d'art, dont l'emblématique Fée électricité de Raoul Dufy, révèlent bien l'ambition de faire étalage des progrès, voire $d u$ progrès, dans une mise en scène finalement commune de la modernitét ${ }^{\text {. }}$ Mais l'Exposition est aussi le réceptacle des enjeux diplomatiques et politiques du temps, ce qu'a retenu la mémoire collective; pendant des mois se font face en se défiant de part en part du Trocadéro, d'un côté l'imposant pavillon allemand surmonté de son aigle impérial, conçu par Albert Speer, de l'autre le pavillon soviétique, "l'Ouvrier et la Kolkhozienne ", comme deux mises en scène de leur puissance belliqueuse. Enfin, si elle fait l'objet de fortes contestations devant son caractère dispendieux, si elle se déroule sur fond de grèves et de manifestations à son ouverture, dans un décor d'édifices et de pavillons parfois inachevés, elle représente néanmoins une vitrine pour la France, qui accueille le monde entier et se place ainsi dans le concert des nations, se muant pour l'occasion en chef 
d'orchestre. Le ton se veut éminemment positiviste et les échanges scientifiques prétendent être le ciment d'une concorde internationale pourtant chancelante.

De nombreuses manifestations scientifiques sont en effet traditionnellement adossées aux expositions et celle de 1937 n'échappe pas à la règle. Au cour de la dense forêt de ces événements - 248 sont annoncés -, allant des plus exotiques aux plus spécialisés, entre journées nationales et grands raouts internationaux, prend place le premier congrès international de Psychiatrie infantile. Il se déroule du 24 juillet au $1^{\text {er }}$ août 1937, logé au cœur d'une quinzaine dite des « congrès intellectuels et d'enseignement " très hétéroclite. Ce congrès tient sa séance inaugurale le 24 juillet après-midi, dans la nouvelle salle des fêtes du Trocadéro, puis ses journées de travail à la Maison de la Chimie, rue SaintDominique, inaugurée en 1934, et se clôt par une courte visite d'agrément sur les bords de la Loire. C'est donc surtout dans le cadre grandiose de la capitale que la psychiatrie infantile tient son premier congrès international. La spécialité est alors balbutiante de part et d'autre du monde médical mais elle possède là une occasion d'acter sa naissance, comme une première étape formelle de son institutionnalisation.

Le congrès de Psychiatrie infantile s'inscrit dans la tradition des congrès scientifiques, très en vogue depuis la fin du $\mathrm{XIX}^{\mathrm{e}}$ siècle. Ceux-ci ont déjà fait l'objet de nombreuses études, qui ont démêlé une grande partie de leurs multiples enjeux ${ }^{5}$. Lieux d'échanges, moments de rencontre entre spécialistes de nombreux pays, ils proposent un état des savoirs à une date donnée, mais ils sont aussi des lieux de frottements entre écoles, courants, pays, pratiques et conceptions scientifiques. Les congrès sont des lieux pour exister, ici pour un collectif de praticiens, autant dans leur propre pays qu'à l'international, dans le même temps aussi pour des individus, soucieux de leur visibilité et de leur promotion, enfin pour une spécialité en tant que telle. Le congrès de juillet 1937 représente bien ce lieu de croisement, qui concentre à lui seul une partie des circulations en cours dans le monde de la psychiatrie infantile. Ce sont en effet plus de quatre-cent congressistes, venus de plus d'une vingtaine de pays, qui convergent vers Paris cet été-là, ayant l'occasion de débattre collectivement pour la première fois de leur objet commun.

Événement éminemment collectif, le congrès n'en est pas moins aussi largement l'œuvre d'un homme: Georges Heuyer (1884-1977), grand ordonnateur de cette manifestation. Au sein de ses archives, la place des documents
5. KaluszYnSki Martine, « Les congrès internationaux d'anthropologie criminelle (1885-1914) », Mil neuf cent. Revue d'histoire intellectuelle (Cahiers Georges Sorel), vol. 7, 1, 1989, p. 59-70; Rasmussen Anne, "Les congrès internationaux liés aux expositions universelles de Paris (18671900) ", Cahiers Georges Sorel, 7, 1989, p. 23-44;

"Les congrès scientifiques internationaux ", Relations internationales, 62/1990; Feuerhahn Wolf, RabaultFeuerhahn Pascale (dir.), "La fabrique internationale de la science. Les congrès scientifiques de 1865 à 1945 ", Revue germanique internationale, 12, 2010. 
6. Boussion Samuel, GUEY Emmanuelle, « Le fonds Georges Heuyer (1884-1977): un xxe siècle scientifique, à l'orée de la psychiatrie infantile et de ses ramifications ", Revue d'histoire de l'enfance "irrégulière ", 12, novembre 2010, p. 215-232. Voir aussi la contribution de Nadine Lefaucheur dans le présent numéro. relatifs au congrès, rassemblés dans quatre boîtes d'époque, bien conservés et soigneusement classés, montre l'importance de ce moment dans son travail au long cours de psychiatre ${ }^{6}$. Sans doute ces archives ressemblent-elles à celles d'autres congrès; elles sont relatives à l'organisation matérielle et scientifique, contenant une correspondance fournie avec les médecins invités ainsi qu'avec les milieux politiques et diplomatiques. Le travail scientifique se dévoile au travers des rapports, rassemblés dans des volumes publiés à la suite du congrès, mais aussi de pièces de correspondance qui rendent compte des oscillations d'une psychiatrie en perpétuelle construction. Mais les archives du congrès de 1937, prises dans l'ensemble du fonds Heuyer et confrontées à d'autres fonds, ceux du ministère de la Santé publique ou des services de l'Exposition, ainsi qu'aux différentes publications professionnelles de la période, permettent aussi de mieux situer une partie de ses enjeux.

En France, le congrès n'est effectivement pas dénué d'enjeux politiques nationaux, ses promoteurs utilisant cette forme pour continuer de peser sur les pouvoirs publics, alors même que le développement de la prophylaxie mentale et la réforme générale de la psychiatrie sont à l'ordre du jour, plus profondément depuis l'avènement du gouvernement de Front populaire en 1936-1937. Il est un événement politique, à partir duquel les organisateurs cherchent à promouvoir leur discipline, mettre en scène leur respectabilité et transformer le congrès en une tribune pour leurs projets de réformes. L'assistance étrangère sert dans ce cadre à éprouver leur attrait international aux yeux des pouvoirs publics et ainsi, le congrès de se transformer en vitrine de la psychiatrie infantile française.

À travers cet événement aux résonances internationales, il s'agit aussi pour la psychiatrie de l'enfant de se séparer du tronc des spécialités dont elle est issue, telles que la psychiatrie générale, la neurologie ainsi que d'autres qui ont contribué à dévoiler l'enfant depuis le début du $\mathrm{xx}^{\mathrm{e}}$ siècle et qui tiennent aussi leur congrès en cet été 1937: psychologie, prophylaxie criminelle et surtout hygiène mentale. Le congrès de Psychiatrie infantile prend place dans une telle dynamique que nombre de participants ont un pied dans plusieurs congrès, notamment celui d'hygiène mentale. Psychiatrie infantile et hygiène mentale comptent également au rang des préoccupations des savants étrangers conviés au congrès, ce qui ne manque pas de dévoiler une partie de ses enjeux politiques, notamment parce que certains d'entre eux représentent des pays où des lois sur la stérilisation, en rapport avec une prophylaxie des pathologies 
mentales, ont été récemment adoptées, rappelant la prégnance de l'eugénisme dans les débats scientifiques et médicaux de la période ${ }^{7}$.

Enfin, le congrès est organisé autour de trois grandes sections pensées comme autant de questions contemporaines et de champs d'intervention: la psychiatrie "générale ", autour des réflexes conditionnels en psychiatrie infantile; la psychiatrie "scolaire ", sur les méthodes d'éducation selon les troubles de l'intelligence et du caractère chez l'enfant; enfin, la psychiatrie "juridique " qui interroge la débilité mentale comme cause de la délinquance juvénile. Le programme balance ainsi entre anciennes questions et tendances nouvelles, les thèmes reposant sur des choix scientifiques mais aussi diplomatiques, en témoignent les invitations répétées à l'endroit des savants soviétiques sur la question de psychiatrie générale, mise au programme pour eux. Plus largement, les archives mettent à jour des questions qui traversent la discipline, par exemple la prégnance du paradigme héréditariste dans l'étiologie de la délinquance juvénile ou encore la préfiguration de nouveaux horizons scientifiques. Au-delà, le congrès pose la question de l'internationalisation de la spécialité. Celle-ci reste à évaluer à travers l'émergence d'une parole commune, dont les vœux émis à l'issue du congrès donnent un premier aperçu.

\section{Georges Heuyer, maître d'ceuvre}

Ce serait au cours d'un congrès de médecine légale en 1932, sans doute le $\mathrm{XVIII}^{\mathrm{e}}$ congrès de Médecine légale de langue française tenu à Paris, que Georges Heuyer aurait caressé pour la première fois le projet d'un congrès international de Psychiatrie infantile. Une idée qui viendrait donc de loin, même si à cette date, il est en convalescence au sanatorium de Sancelmoz, en Haute-Savoie. Certes, ce temps de retrait a pu faire naître chez lui de grands plans pour la psychiatrie infantile, mais plus concrètement, c'est au printemps 1936 que la perspective d'un congrès devient réelle. En mars, le chef du service des congrès de l'Exposition internationale, Abéodat Boissard, professeur de droit, ancien député et figure du christianisme social, accepte la tenue d'un tel événement après une entrevue avec Léon Michaux (1899-1978), ancien interne de Robert Debré et de Georges Guillain, formé à la psychiatrie à l'infirmerie spéciale de la préfecture de Police de Paris par Gaëtan Gatian de Clérambault puis par Heuyer et déjà promu cheville ouvrière du congrès ${ }^{8}$. Une nouvelle entrevue, au mois de mai, cette fois entre Heuyer lui-même et Boissard, scelle l'accord entre
7. Carol Anne, Histoire de l'eugénisme en France. Les médecins et la procréation $x I x^{e}-x x^{\ominus}$ siècle, Paris, Le Seuil, 1995; WEINDLING Paul, L'Hygiène de la race. Hygiène raciale et eugénisme médical en Allemagne, 1870-1932, Paris, La Découverte, 1998, $301 \mathrm{p}$.

8. Université Paris 8 SaintDenis, Fonds Georges Heuyer (FGH) : lettre d'Abéodat Boissard à Georges Heuyer, 24/03/1936. 
9. FGH, lettre de Georges Heuyer à Henri Claude, 13/05/1936.

10. Idem.

11. Coll., Hommage à Georges Heuyer. Pour un humanisme médico-social, Paris, Puf, 1961; Lefaucheur Nadine, « Deux entreprises scientifico-sociales de promotion de l'eugénisme comme fondement des normes en matière de production et de socialisation des enfants: Adolphe Pinard et Georges Heuyer », Vie sociale, mars-avril 1990, p. 61-75; LANG Jean-Louis, Georges Heuyer. Fondateur de la pédo-psychiatrie. Un humaniste $d u x x^{e}$ siècle, Paris, Expansion scientifique publications, 1997;

Boussion, Guey, « Le fonds Georges Heuyer... », op. cit. le comité du congrès de Psychiatrie infantile et le service des congrès quant à l'organisation effective du congrès aux dates fixées?. Entre-temps, Heuyer a pris soin, avant d'accepter la charge d'un tel événement et ainsi mettre fin à ses hésitations, de mesurer sa légitimité auprès de ses maîtres de la génération précédente, dans une révérence tout académique. Les soutiens préalables de Jacques Roubinovitch (1862-1950), Édouard Toulouse (1865-1947), Georges Paul-Boncour (1866-1960), Henri Claude (1869-1945) et Théodore Simon (1873-1961) achèvent effectivement de le convaincre, entraînant pour chacun des remerciements personnels par retour de courrier en même temps qu'une place dans le comité d'honneur du congrès ${ }^{10}$.

Georges Heuyer occupe, à ce stade, une place importante dans le champ de la psychiatrie, plus encore en psychiatrie de l'enfant. Sa biographie compte parmi les mieux connues de la psychiatrie française, en rapport avec sa longévité et ses titres, son intense activité, ses multiples responsabilités exercées dans le champ médico-psychologique jusqu’à ses investissements dans le monde éducatif (scolaire et spécialisé) ${ }^{11}$. Mais il est possible de mettre en relief plusieurs traits marquants, à commencer par son investissement précoce vers la psychiatrie mais aussi vers l'enfance. Passé par la médecine militaire et la neurologie, il s'oriente assez précocement vers la psychiatrie et déjà vers l'étude des pathologies mentales de l'enfance, en témoigne sa thèse de médecine soutenue en 1912 et menée sous la direction d'Ernest Dupré (1862-1921), médecin aliéniste à l'hôpital Sainte-Anne et titulaire de la chaire des maladies mentales et de l'encéphale, chez qui il a réalisé le dernier volet de son internat, qui croise anormalité juvénile, délinquance et école: Enfants anormaux et délinquants juvéniles. Nécessité de l'examen psychiatrique des écoliers. S'il goûte après la première guerre mondiale à d'autres spécialités et affine son choix d'exercice de la médecine, entre la très prisée phtisiologie et la pédiatrie dans le service du Pr Henri Méry (1862-1927) à l'hôpital des Enfants malades, il s'oriente finalement vers la psychiatrie, qui sera pour lui surtout hospitalière et universitaire et non celle du cadre des asiles publics. Il combine alors psychiatrie générale et psychiatrie de l'enfant et multiplie les lieux d'expertise (police, justice, école); en 1920, il est nommé médecin de l'infirmerie spéciale des aliénés près la préfecture de Police et médecin inspecteur des écoles de la Seine, en 1923 médecin des hôpitaux et en 1927 expert près les tribunaux. Surtout, point d'orgue de sa pratique clinique autant que de son rayonnement, il est nommé en 1925 chef de service 
de la clinique annexe de neuropsychiatrie infantile ouverte sous les auspices de la faculté de médecine de Paris au sein du Patronage de l'enfance et de l'adolescence, une institution de placement pour enfants confiés par le tribunal créée en 1895 par le magistrat Henri Rollet, rue de Vaugirard à Paris. Heuyer y accumule les études empiriques sur les enfants en même temps qu'il nourrit de grands projets pour cette spécialité, y formant notamment de nombreux internes et médecins, devenant leur « Patron ", et beaucoup d'entre eux sont propulsés dans les instances d'organisation du congrès de 1937, comme Marguerite Badonnel (1895-1970) ou Jenny Roudinesco (1903-1987), tandis que nombre d'autres grossissent les rangs des participants.

Depuis les années 1920, Heuyer contribue inlassablement à placer la neuropsychiatrie infantile au cœur de l'hygiène mentale de l'enfance et le psychiatre au départ de la chaîne des spécialistes de l'enfance:

\footnotetext{
La méthode neuro-psychiatrique que nous avons indiquée permettra de 《 dépister précocement dans la famille et à l'école ces enfants intellectuellement et moralement anormaux. Elle permettra de les traiter, de les surveiller, de les utiliser en fonction de leurs capacités et de leur nocivité. Elle permettra d'adapter à leur caractère l'éducation et l'instruction, plutôt que de se flatter inconsidérément de les modifier par la pédagogie ${ }^{12}$.
}

Ses activités de recherche, ses publications ainsi que son travail clinique se situent en effet dans la dynamique de la prophylaxie et de l'hygiène mentale. À certains égards, la clinique d'Heuyer prolonge ce qu'Édouard Toulouse a mis en place à l'hôpital Henri Rousselle, lieu-phare de la prophylaxie du département de la Seine ${ }^{13}$. Et à l'instar de Toulouse, son credo repose sur: dépistage précoce et triage en fonction du rendement social, rôle majeur du psychiatre au sein d'une équipe pluri-professionnelle (assistantes de psychologie, assistantes sociales), ouverture de consultations sur le modèle des offices publics d'hygiène sociale pour la lutte contre la tuberculose, de services libres et plus largement volonté d'éviter aux enfants anormaux la prison et l'asile, enfin spécialisation de la prise en charge par la création d'institutions médico-pédagogiques ${ }^{14}$.

Progressivement, au milieu des années 1930, muni d'un curriculum conséquent, Heuyer devient une personnalité majeure de la santé publique en France. D'autant que sa place au cour de la promotion de l'hygiène mentale est confortée par le gouvernement de Front populaire, nommé en juin 1936.
12. HeUYER Georges, « Buts et méthodes de la psychiatrie infantile. Plan d'hygiène mentale de l'enfance ", leçon faite à la Clinique annexe de neuro-psychiatrie infantile, leçon faite le 6/12/1926, p. 10.

13. Huteau Michel, Psychologie, psychiatrie et société sous la Troisième République. La biocratie d'Édouard Toulouse (1865 1947), Paris, L'Harmattan, 2002.

14. HeuYer Georges, " Hygiène mentale de l'enfance ", Le Mouvement sanitaire, 139, novembre 1935, p. 631-635. 
15. Un poste qu'il a déjà tenu en 1932 auprès de Justin Godard.

16. Voir la contribution de Jean-Christophe Coffin dans le présent numéro.

17. Huteau Michel, Psychologie, psychiatrie... p. 218; HENCKES Nicolas Le nouveau monde de la psychiatrie française. Les psychiatres, l'État et la réforme des hôpitaux psychiatriques de l'aprèsguerre aux années 1970. Sociologie. École des hautes études en sciences sociales (EHESS), 2007, https:// tel.archives-ouvertes.fr/ tel-00769780, p. 148
L'enfance déficiente revient fortement à l'ordre du jour. Henri Sellier, socialiste, homme-fort de l'hygiénisme municipal, est nommé ministre de la Santé publique tandis que Suzanne Lacore devient sous-secrétaire d'État à la Protection de l'enfance au sein de ce même ministère. Édouard Toulouse rejoint le cabinet en tant que conseiller technique pour la psychiatrie ${ }^{15}$. Dès 1936 , Sellier institue une Commission pour l'enfance déficiente et en danger moral, présidée par Henri Wallon (1879-1962). Celle-ci devient interministérielle et regroupe alors des représentants de la Santé publique, de l'Éducation nationale et de la Justice, ainsi que des experts, dont Georges Heuyer. Des notes manuscrites de ce dernier relatent les premiers travaux de la commission et son propre champ d'action, à savoir le recensement des enfants déficients du pays. Mais cette vaste entreprise ne résiste pas au départ du gouvernement Blum en 1937, enterrant l'étude en cours en même temps qu'un projet de loi qui en a éclos.

Toulouse et Heuyer partagent nombre d'engagements en psychiatrie, hygiène mentale et eugénisme ${ }^{16}$. Mais surtout, l'agenda professionnel d'Heuyer, apparaît très lié à celui de Toulouse en 1936-1937. Au four et au moulin des préparatifs du congrès de Psychiatrie infantile, Heuyer est lancé en même temps dans les méandres de la succession de Toulouse à l'hôpital Henri-Rousselle. Ce qui pourrait relever de l'épisode de carrière classique au sein d'un milieu médical qui se veut prestigieux et hiérarchisé se révèle au fond moins anecdotique qu'il n'y parait à l'heure du congrès. Les faits sont déjà en partie connus, moins si on les regarde du côté d'Heuyer ${ }^{17}$. Début 1936, 
Toulouse, atteint par la limite d'âge, doit abandonner la direction de l'hôpital, poste sur lequel est détaché Théodore Simon ${ }^{18}$. Les oppositions se réveillent ${ }^{19}$ et Henri Sellier dépose, en tant que conseiller général de la Seine, une requête en annulation devant le Conseil d'État pour abus de pouvoir ${ }^{20}$. Elle est finalement rejetée en mai 1937 mais entre-temps, Sellier, devenu ministre de la Santé publique, a mis Simon à la retraite, ouvrant à nouveau la succession ${ }^{21}$. Déjà pressenti par Toulouse dès le début des années 1930, Heuyer est officiellement candidat, ce qui occupe son esprit à la fin de l'année 1936, livrant cette «bataille» en pleine préparation du congrès ${ }^{22}$. Un court laps de temps, il pense même avoir obtenu ce poste si convoité: " un événement important vient de se produire dans ma vie: je suis nommé directeur de l'hôpital Henri Rousselle comme successeur de M. Toulouse ${ }^{23}$ ». Las, sa joie est de courte durée. Moins d'une semaine plus tard, c'est Georges Génil-Perrin (1882-1964) qui est finalement choisi ${ }^{24}$. À l'évidence, la querelle qui couve depuis le début des années 1930 entre médecins des établissements publics d'aliénés et partisans des services libres, "Toulouse-Jéhovah et son prophète Heuyer ${ }^{25}$ " en tête, est ravivée à cette occasion dans une opposition médecins du cadre vs psychiatres hospitalo-universitaires. Sa déception ravalée, Heuyer annonce qu'il peut désormais se consacrer entièrement à l'organisation du congrès de Psychiatrie infantile, qui n'en revêt que davantage d'enjeux pour lui ${ }^{26} \ldots$

\section{LE CONGRÈS, VITRINE ET TRIBUNE}

\section{DE LA PSYCHIATRIE INFANTILE FRANÇAISE}

Les ambitions d'Heuyer portent les aspirations collectives d'un groupe de professionnels, déterminé à faire émerger la psychiatrie infantile en tant que spécialité et rencontrent alors une commande politique. L'organisation du congrès de 1937 s'inscrit en effet au cour des missions d'hygiène publique arrimées au jeune ministère de la Santé publique. Il opère à la confluence de la montée des politiques de protection de l'enfance et d'hygiène mentale. D'abord, si les dépenses publiques de santé engagées dans l'entre-deux-guerres ont connu une ascension rapide, elles connaissent une nouvelle hausse sensible pour l'année 1937. C'est particulièrement édifiant en matière de protection maternelle et infantile, de lutte contre le cancer mais aussi d'hygiène mentale, ce dernier poste budgétaire devenant même à cette date le plus consistant ${ }^{27}$.
18. Par arrêté du préfet de la Seine en date du 3 février 1936. Cf. L'Aliéniste français, 2, 1936, p. 125.

19. Huteau Michel, Psychologie, psychiatrie..., op. cit., p. 218.

20. Recueil des arrêts du Conseil d'État, 26 mai 1937 (http://gallica.bnf.fr/ ark:/12148/cb343630608/ date).

21. En s'appuyant sur un décret du 6 octobre 1936 qui abaisse l'âge à la retraite des médecins des asiles à soixante-deux ans.

22. FGH: lettre de Georges Heuyer à Jean Dublineau, 22/12/1936.

23. FGH: lettre de Georges Heuyer à Ignace Meyerson, 24/12/1936.

24. Les candidats au poste sont: Génil-Perrin, Heuyer, Por'cher et Vigneron d'Heucqueville. Le concours a lieu le 21 décembre 1936. L'Aliéniste français, 1, 1937, p. 26.

25. Demay Georges, «Psychiatrie d'hôpital et psychiatrie d'asile selon M. Heuyer ", L'Aliéniste français, 1, janvier 1931, p. 274.

26. FGH: lettre de Georges Heuyer à Ignace Meyerson, 31/12/1936.

27. MURARD Lion, ZYLBERMAN Patrick, «Mi-ignoré, mi-méprisé: le ministère de la Santé publique, 1920-1945 ", Les Tribunes de la Santé, 1, 2003, p. 25. 
28. An, Enseignement, CNRS; commissariat général du Salon des arts ménagers, expositions et salons, 19850025/123: lettre d'Henri Sellier à Léon Blum, 26/11/1936.

29. An, Présidence du Conseil, Exposition internationale, F/60/969: subventions proposées au budget 1937.

30. Premier Congrès international de psychiatrie infantile, Paris, SILIC, 1937, p. 29.
Ensuite, si le gouvernement de Front populaire hérite de la mise en œuvre de cette Exposition, qu'il n'a pas initiée ni soutenue, il s'emploie à en faire un succès. Les congrès médicaux en sont une occasion. Le ministère de la Santé publique est très attentif à la bonne tenue des manifestations médicales, comme en témoignent plusieurs lettres de Sellier à Blum dès 1936, l’avertissant par exemple de l'attribution de subventions à « de pseudo-groupements sans qualité ni autorité, à ceux qui y dissimulent les objectifs de publicité commerciale ou à ceux dont le prétexte ne paraît pas extrêmement sérieux ${ }^{28}$ ". Vingt-cinq congrès médicaux figurent au programme de l'Exposition, soit $10 \%$ de l'ensemble. Preuve de l'intérêt manifesté pour la psychiatrie infantile, ce congrès est le mieux doté en subvention, à hauteur de 50000 francs, soit autant que les Journées internationales de chirurgie et les Journées médicales françaises, mais bien plus que les congrès internationaux de Neurologie (30000 francs), d'Hygiène mentale (20 000 francs) ou encore de Médecine légale (10 000 francs) ${ }^{29}$.

Ainsi encouragée, la préparation du congrès débute à l'été 1936, ce dont témoigne le lancement d'une correspondance qui devient abondante. Sans trop d'efforts, le comité d'organisation obtient le haut-patronage de la Présidence de la République, ainsi que celui des segments ministériels concernés: Santé publique, Éducation nationale, Justice, sous-secrétariat d'État à la Protection de l'enfance - nouveauté de la période - et Affaires étrangères pour cause de dimension internationale. Lors du congrès, la présence des représentants politiques achève de convaincre de la réussite des organisateurs à susciter l'intérêt pour leur discipline. Leurs discours, qui ponctuent les journées de travail, montrent leur intérêt pour la psychiatrie infantile et son inclusion dans les politiques de prophylaxie mentale, comme le souligne en ouverture du congrès le Dr Xavier Leclainche (1899-1984), ancien chef de service d'études techniques pour l'Office national d'hygiène sociale et depuis 1935 chef de section au ministère de la Santé publique, pour lequel il développe les services d'hygiène:

On peut dire que le développement de la psychiatrie infantile, - et celui 《de la science qui en dérive, l'hygiène mentale, - conditionne étroitement le progrès de la prophylaxie mentale, c'est-à-dire le succès de la lutte contre le péril qui menace le plus gravement nos sociétés modernes: le péril mental ${ }^{30}$."

L'éclat politique du congrès est en outre couplé à une certaine pompe, chargée de convaincre de la respectabilité de la nouvelle discipline et d'épater les 
délégations étrangères. Les mondanités se succèdent alors dans un Paris doré aux allures de capitale scientifique, faisant passer en quelques jours les congressistes des salons du ministère des Affaires étrangères à un dîner au vieux café d'Harcourt, en plein Quartier latin, d'un thé donné par la Marquise de Ganay, figure des salons littéraires et philanthropes, jusqu'à une garden-party au Palais de l'Élysée avant que ne soit donné le banquet officiel du congrès dans le cadre majestueux du Pavillon Dauphine en lisière du Bois de Boulogne.

Ce faste accompagne pour l'occasion tout un itinéraire au cœur du savoirfaire français en matière de psychiatrie infantile. Sur le site même des conférences, une exposition permanente est chargée de présenter diverses institutions. Clou de l'exposition, un stand est dédié à la consultation du Dr Heuyer, tandis que d'autres sont consacrés au service pour enfants retardés et instables du Dr Gilbert Robin, à l'institut médico-pédagogique de Berck-plage dirigé par Guy Néron ou encore à la Maison d'observation et de rééducation "La Tutélaire ", d'Issy-les-Moulineaux, dont le médecin-chef est Jenny Roudinesco. Dans ce paysage local, on distingue aussi, "international oblige ", quelques institutions étrangères tel que l'Institut de psychologie de Vienne, dirigé par le $\operatorname{Pr}$ Charlotte Bühler ou encore l'Institut de psychologie de Londres, dirigé par le Dr Marguerite Loewenfeld. Ensuite, l'itinéraire se poursuit in situ, dans des établissements jugés " phares ", qui sont autant de déclinaisons d'une psychiatrie infantile « à la française ». La région parisienne est assez riche d'institutions que presque chaque jour les congressistes sont en visite: la colonie d'enfants de Perray-Vaucluse, à l'internat de perfectionnement d'Asnières, la clinique annexe de neuropsychiatrie infantile du Dr Heuyer, à Paris, l'institut médicopédagogique d'Yvetot, enfin l'École Théophile Roussel de Montesson, rebaptisée " institut médico-pédagogique " alors même qu’elle reçoit des enfants délinquants et «moralement abandonnés ». Ce sont toutes des institutions spécialisées publiques, ne recevant que des enfants, car ministère de la Santé et conseil général de la Seine voient d'un mauvais œil la visite d'établissements de santé privés, pourtant nombreux ${ }^{31}$. Surtout, toutes représentent bien la direction prise par la psychiatrie infantile à travers un faisceau d'institutions en marge des asiles - seule la colonie d'enfants du Perray est annexée à un hôpital psychiatrique - et au sein desquelles s'étend l'expertise médicale de l'enfance ${ }^{32}$.

Ce panorama contribue à transformer le congrès en tribune pour les psychiatres. Celui-ci doit en effet donner de l'écho aux projets des promoteurs
31. FGH: lettre de Georges Heuyer à G. d'Heucqueville, $7 / 05 / 1937$

32. Voir la contribution de Mathias Gardet dans le présent numéro. 
33. Von Bueltzingsloewen Isabelle, «Réalité et perspectives de la médicalisation de la folie dans la France de l'entre-deux-guerres », Genèses, 82, mars 2011 , p. 52-74.

34. HeuYER, « Hygiène mentale... », p. 631-635.

35. An, Services communs à la Santé et au Travail, Office national d'hygiène sociale, 19760145/76: Circulaire du ministère de la Santé publique, 23/11/1936, dans « Liste des établissements publics ou privés pour enfants arriérés ou anormaux éducables, dressée par l'Ocов (Office central des œuvres de bienfaisance) », 1937.

36. HeNCKES, Le nouveau monde...

37. FGH: lettre du secrétaire général du Conseil supérieur de l'Assistance publique à Georges Heuyer, $12 / 07 / 1937$. des services libres, de l'hygiène mentale et de la constitution d'un faisceau d'institutions spécialisées (œuvres de rééducation, instituts médico-pédagogiques, écoles de perfectionnement), et au-delà d'attirer vers la psychiatrie une clientèle qui échappait jusque-là à son influence, à commencer par les enfants dits " anormaux ${ }^{33}$ ". C'est ce qu'Heuyer défend depuis les années 1920, appelant de ses vœux la création de consultations de neuropsychiatrie infantile dans les hôpitaux d'enfants, la spécialisation des médecins, une collaboration entre ces consultations et le réseau des institutions spécialisées ${ }^{34}$. Un projet qui ne cesse de prendre de l'épaisseur durant les années 1930, peut-être aussi parce qu'une population plus importante est appelée à entrer dans les circuits de remboursements des frais médicaux, avec le développement des assurances sociales, instituées depuis 1928, mais aussi au bénéfice de l'Assistance médicale gratuite (AмG) depuis une circulaire de $1936^{35}$.

Tout un argumentaire se déploie à cette période sur la nécessaire réforme du système psychiatrique ${ }^{36}$, notamment son versant prophylactique. Beaucoup de psychiatres s'accordent pour trouver la loi de 1838 périmée et limitée. En février 1937, un rapport est déposé au Conseil supérieur de l'Assistance publique sur l'organisation de la prophylaxie des troubles mentaux dans le cadre départemental et ses rédacteurs ont pris soin de l'envoyer à Georges Heuyer en vue de la tenue des prochains congrès internationaux de juillet ${ }^{37}$. Rédigé par Charles Haye, alors directeur-adjoint de l'hygiène et de l'assistance au ministère de la Santé publique, et Jean Lauzier, médecin-chef de l'asile d'aliénés de Clermont, ce rapport spécifie que la loi de 1838 est restée muette sur certaines catégories de malades. L'idée générale est de constituer « l'hôpital psychiatrique moderne ", sous la forme d'une nouvelle prise en charge hors-les-murs de l'asile, par le biais des consultations externes et des services libres. Dans ce domaine, l'assistance psychiatrique de l'enfance fait l'objet d'une attention particulière, qui doit insister sur une importante prophylaxie en milieu scolaire, le développement d'une assistance aux enfants anormaux (développement de la puériculture, lutte contre la tuberculose, colonies de vacances, examens fréquents des enfants, instituts d'orientation professionnelle pour les enfants postscolaires), car est-il répété: « de celle-ci dépend la réelle prophylaxie des maladies mentales et de la criminalité chez l'adulte ":

Jusqu'ici la lutte pour la prophylaxie mentale, bien qu'en progrès vers la $\ll$ victoire, n'a pas encore conquis entièrement la faveur des pouvoirs publics 
et est restée à cet égard sur un plan inférieur par rapport aux moyens engagés contre d'autres fléaux sociaux. Alors que contre la tuberculose il y a près de 60 millions inscrits annuellement au budget de l'État, 15 millions contre le cancer, la prophylaxie mentale n'est pas encore dotée. Or, les maladies mentales, soignées précocement, sont aussi curables que les maladies physiques et la prophylaxie leur est applicable au même titre, avec la possibilité des mêmes résultats positifs si l'on disposait des crédits suffisants ${ }^{38}$. "

La pression des milieux psychiatriques est forte, qui n'ont de cesse de réclamer des moyens à la hauteur des projets ambitieux qu'ils ont élaborés, tandis que l'enfance devient un objet d'attention renouvelée dans les années 1930. Heuyer ne dit pas autre chose en 1938 au sujet des enfants déficients, muni de sa nouvelle aura au sortir du congrès:

En tout cas, puisqu'il faut qu'ils vivent, il faut s'efforcer à ce qu'ils soient une 《moindre charge pour la société [...] nous souhaitons que le Gouvernement mette chaque année dans les sabots des gosses arriérés un nombre de millions suffisant pour réaliser progressivement le projet de loi établi il y a deux ans par la Commission interministérielle de l'enfance déficiente ${ }^{39}$."

\section{UN CONGRÈS SANS TRADITION?}

Psychiatrie infantile, année zéro? Le congrès porte le titre de "premier congrès international de Psychiatrie infantile ». Mais les frontières de la spécialité sont encore mouvantes. L'enfance reste en effet un sous-champ de la psychiatrie. Heuyer lui-même conserve une pratique auprès des adultes, à l'infirmerie spéciale de la préfecture de Police ou au tribunal par exemple. La psychiatrie infantile est aussi liée au cadre asilaire, principal lieu d'exercice de la psychiatrie, ainsi qu’à la neurologie, qui en a été une matrice, ce que rappelle la clinique d'Heuyer, dite de "neuro-psychiatrie infantile». Avant même l'ouverture du congrès, le terme de "psychiatrie infantile " fait figure de nouveauté pour certains médecins, preuve d'une spécialité encore instable. Le Pr Franz Hamburger (1874-1954), qui occupe la chaire de pédiatrie à Vienne, lui préfère celui de " psychopathologie ». Heuyer lui explique que " psychiatrie infantile " renvoie au sens plus large de " psychopathologies de l'enfance " dont l'étude comprend oligophrénies, démences infantiles, troubles du caractère,
38. FGH: HAYE Charles, LAUZIER Jean, «L'hygiène et la prophylaxie mentales bases de l'assistance psychiatrique ", Rapport au Conseil supérieur de l'Assistance publique, février 1937.

39. FGH: HeUYER Georges, "L'enfance déficiente ", article envoyé au Miroir illustré, août 1938. 
40. FGH, lettre de Georges Heuyer à Moritz Tramer, 18/05/1937.

41. «Discours inaugural de M. le Docteur Heuyer ", Premier Congrès..., op. cit., p. 37.

psychopathies de l'enfance ${ }^{40}$. Ce choix n'est pourtant pas une révolution terminologique ni épistémologique puisque dès 1934, le suisse Moritz Tramer a commencé à publier la Zeitschrift für Kinderpsychiatrie, tandis qu'outreAtlantique, Léo Kanner, de la John Hopkins Medical School de Baltimore, publie en 1935 Child psychiatry.

La discussion renvoie en fait à la place du psychiatre parmi les spécialistes de l'enfance. Une grande partie du discours inaugural de Georges Heuyer y est d'ailleurs consacrée, manière de contrer les objections venues d'autres psychiatres, de pédiatres, de psychologues, qui tous ont aussi à s'occuper d'enfants et de leur développement psychique, tout en cherchant à circonscrire le rayon d'action de la psychiatrie de l'enfant. Franz Hamburger imagine celle-ci être une branche de la pédiatrie, d'autant que selon lui le regard psychiatrique accentue la médicalisation de l'enfance et de ses pathologies, un point de vue partagé par les psychologues. Le débat est d'autant plus vif qu'en France, la spécialité pédiatrique est elle-même en cours de reconnaissance, sous l'impulsion de Robert Debré. Alors, Heuyer se fait plus offensif quand il pose les cadres de la psychiatrie infantile, tissant aussi une démarcation selon les âges. Si l'enfance du premier âge est du domaine du pédiatre, il en irait autrement à l'adolescence:

Mais plus tard, à la puberté, lorsque l'enfant a quitté l'école, s'il est un débile

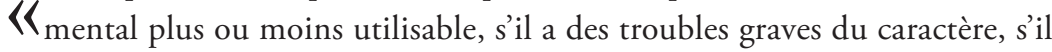
devient un dément précoce, il appartient alors entièrement au psychiatre. »

Il réalise ce même travail de délimitation par rapport aux pédagogues:

Dans toutes les questions qui relèvent de la médico-pédagogie, qu'il s’agisse 《de débiles mentaux ou de déséquilibrés du caractère, les méthodes éducatives doivent être fondées sur les constatations médicales et psychiatriques. Il ne viendrait à l'esprit de personne de demander à un professeur de gymnastique de redresser une scoliose sans les indications précises de l'orthopédiste ${ }^{41}$. "

Une distinction de fait bien réelle puisque bien que "sans tradition », le congrès réunit une large assemblée de doctes médecins, comme le souligne Heuyer dans son discours, «l'élite des psychiatres qui, dans le monde entier, s'occupent de l'assistance, de l'éducation et du traitement des troubles de l'in- 
telligence et du caractère chez l'enfant ». Malgré le dessein initial d'ouvrir ce congrès, il reste le pré-carré des médecins, au grand dam de cet instituteur:

Je me suis inscrit au congrès de Psychiatrie infantile pensant qu'il était destiné
autant aux docteurs qu'aux éducateurs; excusez-moi docteur mais je crois que
je me suis fourvoyé. [...] Qu'ai-je à faire moi, modeste instituteur dans cette
assemblée élégante ${ }^{42}$ ?»

Bien plus ténue est en revanche la frontière avec certaines spécialités qui tiennent leur congrès au même moment et pour qui l'enfance est un objet majeur. Heuyer a ainsi imaginé mettre sur pied une « quinzaine internationale psychiatrique » à l'été 1937, embrassant psychologie, psychiatrie infantile, hygiène mentale, voire prophylaxie criminelle, qu'il estime complémentaires dans le traitement de l'enfance anormale et qui forgent une certaine unité de l'enfance déficiente. Mais en raison de contraintes d'organisation autant que de jeux de pouvoir et de préséance, ces congrès sont finalement programmés sensiblement aux mêmes dates, malgré des réunions d'harmonisation. Jusqu'au bout, la complémentarité entre psychiatrie infantile et hygiène mentale reste chancelante ${ }^{43}$. Pourtant, c'est bien avec le congrès d'Hygiène mentale que la connexion est la plus forte, d'autant que celui-ci dispose de sa propre section d'hygiène mentale infantile, tandis que son objet même, la prophylaxie des troubles mentaux, l'amène naturellement sur le terrain des pathologies de l'enfance. Aussi, les congrès respectifs se succèdent afin de permettre la participation aux deux événements, notamment des savants étrangers, ce qu'Heuyer ne manque jamais de rappeler dans ses courriers afin de mieux mobiliser. Au moment de lancer les invitations, il puise même dans son carnet d'adresses constitué au gré des réunions et congrès d'hygiène mentale. On le sait peu, mais il a en effet participé au premier congrès international d'Hygiène mentale à Washington en 1930. Il en profite alors pour rester un mois aux États-Unis effectuer ce qu'il appelle son "Psychiatric Tour », duquel il rentre très impressionné par les Child Guidance Clinics et plus généralement par l'organisation de l'hygiène mentale, tandis qu'il a gardé des liens avec certains médecins, comme les Dr Plant et Robinson de Newark ${ }^{44}$.

Sept ans plus tard, les contacts tissés à cette occasion sont si denses qu'Heuyer envoie une invitation à rejoindre le comité d'honneur de son congrès à "vingtsix représentants des ligues d'hygiène mentale au congrès de Washington ",

\author{
42. FGH: lettre d'un institu- \\ teur au comité d'organisa- \\ tion, 12/07/1937. \\ 43. FGH: lettre de Georges \\ Heuyer à Robert-Henri \\ Hazemann, 19/02/1937. \\ 44. HeuYer Georges, \\ "L'hygiène mentale aux \\ États-Unis ", La Semaine \\ des hôpitaux de Paris, \\ 15 janvier 1931, p. 3-15.
}


45. FGH: lettre de Georges Heuyer à Pierre Janet, 30/10/1936

46. Voir la contribution de Volker Roelcke dans le présent numéro

47. FGH: lettre de Georges Heuyer à Clifford Beers, 02/11/1936

48. Avec le Dr Carrère, « Hérédité psychopathique polymorphe »; avec Mlle Courthial, « Constitution et eugénique ».

selon des annotations en marge d'un courrier ${ }^{45}$. Ces contacts s'étoffent par la suite; en 1932, lors d'une réunion internationale de prophylaxie et d'hygiène mentale à Paris, Heuyer retrouve le Hollandais Bouman, les Belges Verwaeck et Vermeylen, les Allemands Weygandt, Rüdin et Sommer, l'Espagnol Germain ou le Norvégien Evensen, qu'il invite ensuite à figurer au comité d'honneur du congrès de Psychiatrie infantile. Si bien qu'au final, à l'image d'Ernst Rüdin ${ }^{46}$, bien d'autres participants ont un pied dans les instances des deux congrès: Auguste Ley, Klaas Herman Bouman et Henri Claude sont à la fois membres du bureau du congrès d'Hygiène mentale et présidents d'honneur du congrès de Psychiatrie infantile; Pacheco da Silva et Plinio Olinto, du Brésil, membres de la Société brésilienne d'hygiène mentale, de même que José Belbey, de BuenosAires, ou encore Arthur H. Ruggles, du Butler Hospital de Providence, Adolf Meyer, du John Hopkins Hospital de Baltimore, sont membres du bureau du congrès d'Hygiène mentale mais aussi membres du comité d'honneur du congrès de Psychiatrie infantile. Heuyer est même tenté d'enfreindre la règle voulant que le comité d'honneur soit réservé à des médecins en priant Clifford Beers, secrétaire général de la Ligue internationale d'hygiène mentale, d'en faire partie, invitation que celui-ci décline pour mieux se concentrer sur le congrès d'Hygiène mentale ${ }^{47}$.

De son côté, Heuyer est aussi membre du comité d'organisation du congrès d'Hygiène mentale, mais il n'y met aucune force. Son programme le conduit vers la première réunion de la Fédération des sociétés latines d'eugénique, du $1^{\text {er }}$ au 3 août 1937, à la faculté de médecine de Paris. Elle est logée au coeur des « journées de l'eugénisme » et suit le congrès international de la Population. Heuyer s'y montre très actif et présente deux communications ${ }^{48}$. C'est aussi un moment éminemment politique, puisqu'un an plus tôt, en juillet 1936, Heuyer a été l'émissaire du gouvernement français, ainsi que le représentant de la Société française d'eugénique, à la conférence de la Fédération internationale des sociétés d'eugénique, sur les bords de la mer du Nord, à Scheveningen aux Pays-Bas. Certes impressionné par les travaux allemands de génétique exposés à cette occasion, Heuyer enseigne à Henri Sellier que l'enjeu a été double: éviter que le prochain congrès international d'Eugénique se tienne à Berlin en 1937 et empêcher la réélection de Rüdin à la tête de la Fédération. Les lois et pratiques de stérilisation sont au cœur des préoccupations des eugénistes, mais Heuyer affirme que si intéressante soit-elle, la politique allemande a pris un tour 
politique et dépassé son strict caractère scientifique, faisant sans doute référence aux modifications récentes de la loi de $1933^{49}$. Objectifs atteints finalement, selon Heuyer, qui s'attribue le rôle majeur dans ce nouveau rapport de forces; le congrès n'aura pas de dimension internationale et Rüdin doit accepter de laisser la place, c'est le Suédois Torsten Sjögren qui est élu à l'unanimité ${ }^{50}$.

Georges Heuyer expose par ailleurs à plusieurs reprises que le congrès de Psychiatrie infantile n'a pas de tradition. S'il existe bien une tradition de congrès internationaux de psychiatrie, ils ont cessé depuis la première guerre mondiale. La plupart des médecins présents à Paris en 1937 sont néanmoins rompus aux congrès internationaux, passages obligés de la science médicale. On les voit ainsi arpenter au fil des ans les congrès internationaux de neurologie et de psychiatrie, dans leurs déclinaisons géographiques et linguistiques par exemple. Ils fréquentent aussi les congrès d'autres spécialités plus installées: la pédiatrie tient son $\mathrm{IV}^{\mathrm{e}}$ congrès en septembre 1937 à Rome; la psychologie en est en juillet 1937 à son $\mathrm{XI}^{\mathrm{e}}$ congrès international; l'hygiène mentale a inauguré ses congrès en 1930 à Washington avant d'organiser son second en juillet 1937 à Paris...

Au-delà de ces réseaux, Heuyer s'appuie sur ses connaissances des réseaux psychiatriques de différents pays. Depuis les années 1920, les circulations internationales dans le champ de la psychiatrie se sont en effet intensifiées ${ }^{51}$. La présence discrète au congrès de Psychiatrie infantile comme à celui d'Hygiène mentale de Daniel P. O’Brien, représentant de la Fondation Rockefeller en Europe, rappelle l'investissement croissant de la philanthropie américaine en psychiatrie ${ }^{52}$ ainsi que l'internationalisation du champ. L'heure est aussi à un intérêt renouvelé pour les systèmes psychiatriques étrangers, comme en témoigne par exemple la revue Annales médico-psychologiques, qui ouvre en 1935-1937 une rubrique exposant les tendances contemporaines de la psychiatrie à partir de réalisations nationales qui compteraient parmi les plus avancées dans ce domaine, qui toutes font une place à l'enfance dite « anormale».

Le processus de sélection des participants au congrès, qui s'ouvre en même temps que s'élabore un programme, révèle tout un travail relationnel, scientifique et diplomatique, certes selon une stratégie échelonnée mais aussi dans l'idée d'accumuler au maximum les noms et les pays représentés. Le premier temps est dédié à la composition d'un comité d'honneur, on l'a vu. Membres éminents et au plus près du champ de la psychiatrie infantile, ceux-ci sont en

\begin{abstract}
49. Massin Benoît, «Stérilisation et contrôle médico-étatique des naissances en Allemagne nazie (1933-1945). La mise en pratique de l'Utopie biomédicale », Giamı Alain, LeRIDON Henri (dir.), Les enjeux de la stérilisation, Paris, INSERM, 2000, p. 63-122.
\end{abstract}

\section{Archives municipales de Suresnes, fonds Cabinet ministériel Henri Sellier, carton D660 : rapport de Georges Heuyer à Henri Sellier, 24/07/1936.}

51. ROELCKE Volker, WEINDLING Paul J., Westwood Louise (dir.), International Relations in Psychiatry. Britain, Germany and the United States to World War II, Rochester, University of Rochester Press, 2010.

52. Kriege John, Rausch Helge (dir.), American Foundations and the Coproduction of World Order in the Twentieth Century, Göttingen, Vandenhoeck \& Ruprecht, 2012. À partir de 1932-1933, la psychiatrie devient le point principal, aux côtés de la neurophysiologie, des investissements de la Medical Sciences Division de la Fondation Rockefeller. Voir <https:// www.rockefellerfoundation.org/app/uploads/ Annual-Report> 
53. Voir la contribution de Martine Ruchat dans le présent numéro. outre chargés par Heuyer d'obtenir le concours des médecins les plus qualifiés dans les questions à l'ordre du jour, qu'il ne connaît pas nécessairement dans un monde psychiatrique aux multiples recoins. Une fois ce comité sur pied, Heuyer constitue ensuite un "comité de propagande ", chargé d'assurer dans chaque pays publicité et relais des travaux, qui se veut volontairement pléthorique. Enfin, il invite certains membres ou participants, appelés à être physiquement présents, dont certains ont l'honneur de voir leurs travaux présentés. Au final, 415 membres adhérents sont inscrits, représentant 26 pays. S’il s'agit d'un congrès international, la comptabilité des adhérents (participants) se fait pourtant sous la bannière nationale de chacun. Hormis les invités personnels de Georges Heuyer et du comité d'organisation, ce sont donc des délégations officielles qui se présentent à Paris, bien souvent aussi avec l'idée d'intéresser leur propre gouvernement à la discipline, qui participe même d'une certaine façon à la nomination des membres officiels ${ }^{53}$. Heuyer entretient lui-même cette distinction nationale dans son introduction quand il réalise un tour d'horizon historique et géographique de la discipline, dans lequel chaque grande puissance est présentée, de manière parfois assez grossière, selon son axe fort: la pédagogie en Suisse, la psychanalyse en Autriche, l'école pavlovienne en Union soviétique, les tests aux États-Unis, la guidance infantile en Grande-Bretagne...

Cela a pour effet de renationaliser les échanges, mais nous permet aussi de dresser une cartographie des puissances et des territoires de ce champ, presque une géopolitique. Dans cet ensemble, la France, pays hôte, compte de manière évidente le plus grand nombre de représentants présents, en raison de l'aspect local, de son nombre déjà important de spécialistes des anormalités juvéniles ainsi que du souci de participer et de se montrer lors de cet événement majeur réunissant les élites médicales du pays. Si l'on se réfère aux membres du comité d'honneur ainsi qu'aux membres effectivement présents (adhérents), la délégation allemande est la seconde en termes d'importance. Outre la proximité géographique, c'est surtout que l'heure n'est plus au boycott des scientifiques allemands comme dans les années post-guerre. Mieux même, c'est Ernst Rüdin, directeur du Kaiser Wilhelm Institut de Munich et figure majeure de la psychiatrie du Reich, qui a l'honneur, non seulement de mener sa délégation, mais aussi d'être le porte-parole des délégations étrangères, sans que l'on connaisse le processus de nomination à ce poste. Cela lui vaut de faire un discours suave et plein de diplomatie, célébrant l'hospitalité de ses hôtes, le rôle précurseur 
de la France en psychiatrie infantile ainsi que d'en appeler aux débats et à la compréhension mutuelle, gages de paix et de civilisation...

Cette puissance allemande met en lumière, par contraste, l'absence de représentants soviétiques. Malgré les efforts d'Heuyer, parce qu'il a inscrit à l'ordre du jour l'étude des réflexes conditionnels chez l'enfant, directement inspirée des travaux des physiologistes de l'école pavlovienne, même s'il a pu compter sur le soutien d'Alexandre Roubakine (1889-1979) pour établir le contact ${ }^{54}$, la géopolitique et le processus de purges dans le pays ont raison de la présence de ces médecins. Du même coup, l'autre grand pôle dominant est la psychiatrie infantile des États-Unis. La place centrale accordée à l'enfant dans le maillage d'hygiène mentale est manifestement un point qu'Heuyer souhaiterait transposer en France, étant entendu selon lui que les pathologies et défauts des adultes sont bien souvent des développements de constitutions défectueuses rencontrées chez l'enfant, d'où ses efforts à leur endroit ${ }^{55}$. D'éminentes figures du paysage médico-psychologique d'outre-Atlantique ont fait le voyage: Frederick Patry de New York, Arnold Gesell de la Psychoclinic de Yale ou encore Arthur Ruggles de Providence, sans oublier William Healy et Lauretta Bonner, de la Judge Baker Foundation de Boston ${ }^{56}$. Enfin, si l'on se reporte au nombre de délégués élevés au rang de rapporteurs, de nombreuses puissances périphériques s'affirment: Grande-Bretagne surtout mais aussi Pays-Bas, Belgique, Suisse, pays Scandinaves, Italie, Espagne et Portugal, ainsi qu'Argentine et Brésil.

\section{L'ENFANCE ANORMALE SOUS EXPERTISE: LES RÔLES DU PSYCHIATRE}

Le choix des thèmes du congrès répond aux problématiques de la psychiatrie infantile du temps, mais aussi aux intérêts des organisateurs. Les sections de travail qui découpent le congrès montrent les directions prises par cette spécialité pour approcher l'enfant. La première d'entre elles est dite " psychiatrie générale ", qui rappelle par opposition à quel point les deux autres seraient "spécialisées ", en l'occurrence "scolaire " et " juridique ». Elle est spécifiquement axée sur «Les réflexes conditionnels en psychiatrie infantile ». Pour Heuyer, il s'agit d'un sujet des plus neufs et resterait méconnu dans ses applications. Ainsi, selon lui, travailler sur les réflexes conditionnels afin d'établir les lois qui les régissent permettrait d'expliquer de nombreux symptômes de maladies mentales. Heuyer rappelle qu’à la suite des expériences de Pavlov, ils sont des " associations anormales physiologiques, artificielles ou accidentelles

\author{
54. Emigré russe échappé \\ des prisons du tsar, \\ Roubakine obtient son \\ titre de médecin à Paris. \\ Représentant du commis- \\ sariat à la Santé publique \\ russe dans la France des \\ années 1920 mais aussi \\ expert de la Société des \\ Nations, boursier de la \\ Fondation Rockefeller, il \\ fait figure d'intermédiaire \\ entre l'Urss et le monde \\ occidental. LAHUSEN Thomas, \\ Solomon Susan, In Search \\ of Roubakine, film $66 \mathrm{~min}$, \\ Chemodan Films, 2012. \\ 55. Heuyer Georges, \\ « L'hygiène mentale... », \\ op. cit. \\ 56. Voir la contribution de \\ Guillaume Périssol dans le \\ présent numéro.
}


57. FGH: lettre de Georges Heuyer à Mac Calman, 20/08/1936.

58. Gemelli Agostino,

«Les réflexes conditionnels en psychiatrie enfantine ", Rapport introductif et rapports de psychiatrie générale, Premier Congrès..., p. 129. Voir la contribution d'Elisabetta Benetti dans le présent numéro.

59. Dufaud Grégory,

"Quel usage des thèses pavloviennes en médecine? Schizophrénie, incertitudes scientifiques et psychiatrie en Union soviétique », Cahiers du monde russe, 1 , 2015, p. 214 mais toujours accidentelles, acquises et conditionnées par les circonstances, et qui sont remarquablement fixes, constantes et automatiques ${ }^{57}$ ». Avant même le début du congrès, cet enthousiasme n'est pourtant pas partagé par tous, loin de là, Mac Calman et Rumke, par exemple, considérant qu'il s'agit là d'un sujet un peu étroit.

Tout à son exploration de nouvelles pistes de compréhension des troubles de l'enfant et particulièrement dans l'optique d'une sélection toujours plus fine et précoce des anormaux, Heuyer entend ainsi porter l'attention des médecins sur les bases physiologiques de la psychiatrie. Il semble découvrir ces orientations de recherche alors même que leurs échos sont déjà nombreux, en URSS certes, mais aussi aux États-Unis et en Europe, comme le rappelle dans sa communication Agostino Gemelli58. En France, les travaux s'agrègent progressivement depuis la traduction de l'ouvrage de Pavlov, Les réflexes conditionnels, en 1927, par exemple dans L'Encéphale ou dans L'Evolution psychiatrique en 1935.

Le thème de psychiatrie générale est sans doute le reflet d'un intérêt renouvelé pour la science soviétique. Heuyer argumente dans chacun de ses courriers sur le fait qu'il a placé cette question au programme justement pour que ces savants fassent connaittre leurs travaux. Derrière cette invite pressante, sans doute peut-on repérer un philosoviétisme personnel - facilité par le fait que l'épouse d'Heuyer est russe - autant que collectif car répandu dans les sphères intellectuelles et scientifiques françaises, surtout depuis l'avènement du Front populaire, comme l'illustrent les nombreux voyages en Union soviétique, malgré le hiatus récent de la publication du récit de voyage d'André Gide. Le souhait d'Heuyer est si impératif qu'il montre une certaine méconnaissance des tensions entre physiologistes et psychiatres en Russie, comme en témoigne son invitation simultanée à Ivanov-Smolensky, pur disciple de Pavlov, et Gourevitch, principal contempteur de la physiologie, qui accuse le premier d'avoir « des positions théoriques mensongères ${ }^{59}$ \%. Enfin, un argument décisif de cette sollicitation relève sans doute du politique, la présence soviétique devant offrir un contrepoint à la domination allemande, même si cela n’est jamais exprimé ainsi.

L'intérêt d'Heuyer repose aussi sur une question scientifique et un intérêt pour les travaux de Nicolas Oseretsky, de l'Institut de Léningrad, sur les troubles psychomoteurs. Depuis 1935, L'Evolution psychiatrique s'intéresse à ses recherches et notamment au développement d'un instrument de mesure des capacités psychomotrices. Heuyer lui-même a introduit dans sa clinique 
le test créé par Oseretsky afin de mieux dépister les troubles psychomoteurs. Les archives montrent l'espoir mis sur ces techniques d'investigation, considérées comme porteuses d'avenir pour l'explication de certains symptômes de névroses et de psychoses, surtout pour déterminer les insuffisances motrices chez les enfants au développement intellectuel normal. Las, à l'heure où les grandes purges ont débuté, les savants soviétiques sont absents des festivités ${ }^{60}$, tandis qu'Oseretsky avance, lui, un séjour en sanatorium au moment de décliner l'invitation. Au final, Heuyer se montre mitigé devant les travaux de cette section, amputée des Soviétiques, déplorant du coup le manque d'applications pratiques et techniques ainsi que le peu d'instrumentation et de technique exportable proposées.

Les travaux de psychiatrie scolaire sont beaucoup plus classiques. Ils portent le sous-titre de " méthodes d'éducation selon les troubles de l'intelligence et du caractère chez l'enfant ", qui ramènent à la question du dépistage et de la mesure des retards et déviances en milieu scolaire mais aussi à l'éducation appropriée pour les enfants ainsi détectés. L'école figure depuis longtemps comme un poste privilégié pour le dépistage des enfants anormaux et de nombreux pays ont adopté une législation favorisant une éducation spéciale. D’une manière générale, les psychiatres investissent le champ de la pédagogie de l'enfance déficiente, en témoigne par exemple le courant de pédagogie curative (Heilpädagogik) en Suisse ou en Allemagne. Heuyer lui-même participe au comité de rédaction de la Zeitschrift für Kinderforschung aux côtés d'Ibrahim, Villinger ou encore Hanselmann. Alors, si l'éducation des enfants déficients semble faire consensus pour les enfants souffrant de troubles de l'intelligence, autour de méthodes concrètes de lecture, de mise au travail ou encore du rôle de la gymnastique, il n'en est pas de même des enfants souffrant de troubles du caractère, qui sont visiblement l'objet de discussions. L'enjeu est important autour de cette catégorie d'enfants qui manifestent des symptômes d'agressivité, d'instabilité, d'opposition, etc., qui se consolide en France dans les années 1930. Certes, intelligence normale et caractère équilibré doivent s'accorder pour garantir à l'enfant des chances de s'accomplir, lit-on alors, mais Heuyer fait du caractère une variable prépondérante:

Une intelligence moyenne, même un peu inférieure à la normale, si elle 《est soutenue par une volonté solide, donne un meilleur rendement qu'une

\author{
60. Alexandre Luria \\ fait néanmoins parve- \\ nir son rapport: «Vues \\ psychologiques sur le \\ développement des états \\ oligophrènes ».
}


61. Heuyer Georges, "L'hygiène mentale aux États-Unis », La Presse médicale, 54, 9 août 1930,

p. 1.

62. « Discours de M. le Docteur Georges Heuyer », Premier Congrès..., p. 231.

63. Heuyer Georges, « La psychanalyse », Supplément au Journal de médecine, 10 août 1924, p. 533-546.

64. FGH: lettre de Georges Heuyer à Joseph Friedjung, 22/09/1936.

65. FGH: lettre de Sophie Morgenstern à Georges Heuyer, 31/07/1937. intelligence vive et développée que contrarient des tendances à l'instabilité, des goûts excessifs au plaisir ou une émotivité exagérée ${ }^{61}$. "

Cette section a visiblement concentré les points de vue d'orientation psychanalytique, à travers les rapports de Frederick Patry, Emmanuel Miller ou Pierre Mâle. Mais Heuyer ne s'en cache pas quand lui revient le mot de la fin, la psychanalyse n'est pas applicable sur le plan thérapeutique dans un pays qui a fait le choix des internats et de la rééducation collective:

Sans doute, les psychanalystes peuvent nous dire qu'ils connaissent les 《méthodes à employer et qu’un traitement psychanalytique régulier et longtemps soutenu permet le redressement des caractères les plus difficiles. Cette affirmation peut être partiellement exacte quand il s'agit d'un traitement individuel des troubles caractériels qui gênent l'adaptation du sujet au milieu familial. Mais, dans les établissements qui réunissent des enfants délinquants ou des enfants difficiles que leur instabilité ou leur déséquilibre n’a pas permis de laisser dans un milieu normal, les méthodes psychanalytiques ne sont plus de mise. Il s'agit d'appliquer des méthodes d'éducation collective, de même que dans les établissements pour enfants arriérés, il faut des méthodes d'enseignement collectif ${ }^{62}$.»

D’une manière générale, la psychanalyse, qui connaît un frémissement en France et dans d'autres pays et qui a connu un temps les faveurs de Georges Heuyer ${ }^{63}$, semble désormais barrée par de sérieuses réserves. Son caractère théorique est mis en avant, autant que le fait qu'elle peut être exercée par des nonmédecins; il est d'ailleurs refusé pour cette raison à Anna Freud de siéger au comité d'honneur du congrès ${ }^{64}$. Réserves qu'a peut-être bravées la psychanalyste d'enfants Sophie Morgenstern, qui se confond en excuses auprès d'Heuyer pour avoir fait passer sur le congrès " involontairement un tout petit nuage (5) $^{6}$, dont on ne connaît pas l'origine...

L'intérêt pour la question de psychiatrie juridique, «la débilité mentale comme cause de délinquance infantile ", témoigne de préoccupations anciennes et William Healy, membre du comité d'honneur du congrès mais aussi rapporteur de la section, estime de prime abord que la question est épuisée et manifeste son scepticisme auprès d'Heuyer, rejetant en outre la notion d'intelligence au profit d'autres facteurs. Cette étiologie rebattue au moins depuis le début 
du $\mathrm{Xx}^{\mathrm{e}}$ siècle vise à étudier le rôle de la débilité mentale dans la délinquance juvénile, ainsi déterminer le discernement de l'enfant et sa responsabilité. Car là encore le contexte renforce l'actualité de la question, tandis que la clinique médico-sociale de l'enfance délinquante s'accentue, peu de temps après la dépénalisation du vagabondage par les décrets-lois de 1935, alors que la campagne de presse contre les « bagnes d'enfants » encore toute chaude a laissé des traces. Le recours à une expertise médico-psychologique systématique de tous les enfants délinquants est présenté comme un outil important de la réforme de la justice des mineurs venant supplanter l'ancien "discernement », tandis que la mesure des facteurs de la délinquance reste le pilier de l'action des psychiatres sur le plan criminologique. Pourtant, à l'heure des bilans, si Heuyer vante une forme de consensus, pour expliquer par exemple que personne ne conteste le fait que nombre d'enfants débiles deviennent délinquants tout en reconnaissant qu'un enfant ne devient pas toujours délinquant parce qu'inintelligent, en revanche la définition de la débilité mentale pose question, puisque pour certains rapporteurs, la débilité mentale groupe à la fois arriération intellectuelle et troubles du caractère.

Ainsi est posée la question d'un langage commun élaboré durant le congrès. Celui-ci se retrouve dans les vœux posés à l'issue de celui-ci. L'un des premiers repose sur un appel à l'internationalisation et à la constitution d'un esprit de corps, par la fondation d'une Société internationale de psychiatrie infantile, notamment chargée d'organiser les congrès. Elle est déjà en discussion avant même le démarrage du congrès de 1937, entre les membres de son futur bureau. Elle est présidée par le Pr Schröder, de Leipzig, où doit se tenir le second congrès international en 1941. Le secrétariat général est confié à Moritz Tramer et son siège est en Suisse, tandis que pour assurer la continuité, Georges Heuyer en est le vice-président. Sur le plan scientifique comme sur celui des applications médico-psychologiques et médico-pédagogiques, les conclusions dessinent les suites du congrès et les orientations d'une discipline en perpétuel ajustement. D’abord, le désir de faire pénétrer la psychiatrie par des méthodes scientifiques de mesure, dans l'idée notamment de trancher la question du rôle de l'hérédité et du milieu dans la constitution de la personnalité de l'enfant, non sans délaisser les conditions sociales comme y invite le $\mathrm{V}^{\mathrm{e}}$ vœu, mais aussi par le biais d'outils toujours plus élaborés d'évaluation à des fins d'orientation des enfants. Par-delà les spécialités développées dans chaque pays, l'idée est aussi de préciser 
les descriptions et d'unifier les termes, comme le souligne le IV vœu en plaidant pour la création d'une commission consacrée à l'établissement d'une nomenclature et d'instruments de mesure internationaux. Enfin, la place du psychiatre est appelée à être renforcée en vertu de son expertise, notamment auprès des enfants délinquants, par la systématisation des examens médico-psychologiques, par la collaboration avec les pédagogues dans les centres médico-pédagogiques mais aussi par la direction d'une observation au long cours des enfants avant toute orientation ou rééducation.

Ainsi, le congrès de 1937 ouvre de multiples perspectives pour la psychiatrie infantile, s'efforçant de bâtir le futur rôle du psychiatre d'enfant dans la société, bien plus loin que la simple pratique clinique, sortant aussi du strict registre des pathologies mentales de l'enfant pour s'avancer vers l'étude et la thérapeutique des comportements juvéniles. Le congrès n’est pas révolutionnaire sur ce plan, car il vient en aval d'un processus engagé depuis les années 1920 mais sans doute le comparatisme à l'œuvre en 1937 permet d'avancer de nouveaux jalons, qui éclosent autour de la seconde guerre mondiale. Ainsi, c'est peu avant ou pendant le conflit que certains des voux issus du congrès se trouvent en partie réalisés en France: extension des dispositifs d'hygiène mentale (consultations neuropsychiatriques, dépistage scolaire...) par circulaires du ministre de la Santé publique Marc Rucart, dont celle du 13 octobre 1937 sur la réorganisation de la prophylaxie mentale, développement de nosographies plus précises, en 1943 notamment, plus généralement une montée en expertise des psychiatres auprès de l'enfance à travers l'observation, qui devient un élément majeur de la praxis en psychiatrie infantile. L'institutionnalisation de la psychiatrie infantile se poursuit alors, même au gré des changements de gouvernement. Elle est marquée en France par la création en 1948 d'une chaire de neuropsychiatrie infantile à la Faculté de Paris, dévolue à Georges Heuyer, comme une reconnaissance et une consécration. Sur le plan international, la guerre empêche la tenue du congrès suivant prévu en 1941 en Allemagne. Plus encore, elle va tarir les circulations antérieures au profit de nouvelles, avec la montée en puissance des pays vainqueurs, tandis que les objets et pratiques vont en sortir modifiés. Le conflit a rebattu les cartes là aussi et c'est à Londres, en 1948, que se déroule finalement le second congrès international de Psychiatrie infantile, plus d'une dizaine d'années plus tard, pendant lesquelles la spécialité s'en est trouvée changée certes, mais affirmée. 